\title{
Internet addiction and criminal phenomenology: an insight on the Social Networking Sites addiction and the online death games
} Internet addiction e fenomenologie criminali: un approfondimento sulla dipendenza da social network e gli online death games Addición a internet y fenomenologías criminales: una profundización de la addición a las redes sociales y los juegos mortales online

\author{
Claudia Colombo, ${ }^{1}$ Monica Calderaro ${ }^{2}$ \\ ${ }^{1}$ Dottoressa Magistrale in Investigazione, Criminalità e Sicurezza Internazionale, Università degli Studi Internazionali \\ di Roma (UNINT); ${ }^{2}$ Grafologa forense, Criminologa, Dottore in Psicologia ad indirizzo clinico, Responsabile Didattico \\ e Docente del Corso di Formazione in Grafologia Forense, Sapienza Università di Roma, Italy
}

\begin{abstract}
With the advent of the virtual world as the counterpart of our daily lives, several concerns emerged. The following paper will focus on the iGeneration, a fragile social group grown up between the online and the offline world, and its chances to develop a technology addiction. Secondly, an insight on the illustrative and extreme scenario of the Blue Whale Challenge, a death challenge emerged on social networking sites, its implications and connection with the real world.
\end{abstract}

\section{RIASSUNTO}

L'avvento del mondo virtuale come controparte delle nostre vite reali ha generato diverse preoccupazioni. Nel seguente elaborato, lo sguardo sarà incentrato sull'iGeneration, categoria fragile cresciuta a cavallo fra i due mondi online e offline, e sulla possibilità dei suoi giovani membri di sviluppare forme di dipendenza dalla tecnologia. Seguirà un approfondimento sul caso estremo della Blue Whale Challenge, sfida mortale nata sui social network, le sue implicazioni e legami col mondo reale.

\section{RESUMEN}

La aparición del mundo digital como contraparte de nuestras vidas reales ha creado diferentes preocupaciones. En el siguiente documento, la reflexión será centrada en la iGeneration, frágil categoría social crecida a caballo entre el mundo online y offline, y en su posibilidad de desarrollar una adicción de la tecnología. Sigue un análisis en profundidad sobre el caso extremo de la Blue Whale Challenge, un mortal desafío surgido en las redes sociales, su implicaciones y conexiones con el mundo real.

\section{Introduzione}

Il 30 settembre 2020 le maggiori testate giornalistiche italiane hanno riportato una tragica notizia riguardante un bambino napoletano di 11 anni morto suicida, plagiato da un fantomatico soggetto con l'aspetto di un inquietante Pippo Disney. Il mostro, che poi si è scoperto essere un personaggio della cui identità si sono serviti diversi utenti dei principali social network, prende il nome di Jonathan Galindo.

Lo sconcerto che ha accompagnato questo caso ha portato a cercare immediatamente le motivazioni dietro il gesto fatale del bambino che, la notte del 29 settembre, si sarebbe gettato dal balcone di casa. Le indagini si sono concentrate sulla possibilità di istigazione al suicidio e che, nei giorni immediatamente precedenti, il bambino fosse entrato in contatto tramite i social con una figura che lo avrebbe minacciato o spaventato a tal punto da fargli commettere l'azione suicidaria. L'ipotesi sembra sostenuta dal messaggio che il bambino ha inviato alla famiglia prima di togliersi la vita, con cui esprime il suo amore per i genitori, fa riferimento alla sua paura e annuncia di dover "seguire l'uomo nero col cappuccio".

È stato proprio il richiamo all'immagine dell'uomo col cappuccio che ha aperto alla possibilità del coinvolgimento del bambino a una sfida della morte legata al fenomeno della Creepypasta, cioè la diffusione di leggende dell'orrore online, che terrorizzano i più giovani fino a trascinarli in un gioco mortale. Il richiamo alla sfida della morte, ha condotto al nome di Jonathan Galindo, un personaggio incappucciato con l'aspetto di un cane antropomorfo, che avrebbe il ruolo di curatore di una nuova death challenge che utilizza i social network come terreno di reclutamento delle giovani vittime.

Il caso Jonathan Galindo è ancora avvolto nel mistero: non si conoscono l'identità, o le identità, degli organizzatori del gioco, esistono poche informazioni sul loro modus operandi e sulle loro tecniche di adescamento, fatto salvo il contatto con le potenziali vittime attraverso il mondo virtuale. Immediato però è stato il collegamento con un'altra sfida mortale che pochi anni fa ha creato un vero e proprio fenomeno di psicosi di massa e isteria mediatica, 
la Blue Whale Challenge. La sfida mortale della balena blu ha coinvolto i giovani di diversi Paesi del mondo, stimolando inchieste giornalistiche e analisi psico-sociali del fenomeno.

Obiettivo del presente elaborato è mettere in luce gli aspetti caratterizzanti degli Online Death Games attraverso l'esempio della Blue Whale Challenge, cercando di illustrare il fenomeno come deriva estrema dell'impatto delle nuove tecnologie comunicative sulla più giovane generazione.

\section{iGeneration: comunicazione e salute mentale}

Ogni epoca storica è condizionata dalle tecnologie comunicative presenti: oggi è innegabile che gran parte delle nostre vite sono legate a internet e alle possibilità di accessibilità, connessione e relazione che è in grado di offrire. L'iGeneration (L.D. Rosen, 2011) è senza dubbio la coorte maggiormente coinvolta in tal senso: l'espressione identifica tutti i soggetti nati dagli anni Novanta a oggi, i quali hanno avuto modo di crescere con le nuove tecnologie comunicative. I membri della nuova generazione non solo sono in grado di realizzare ogni tipo di attività nella dimensione online, ma sono gli unici che hanno avuto modo di costruirsi la propria identità sia nel mondo reale sia nel mondo virtuale offerto da internet e dai social media.

La scelta di questo target di riferimento non è casuale: negli ultimi anni si è registrato un numero sempre maggiore di giovani coinvolti in problemi di salute mentale, dati da un lato dalla fragilità psicologica tipica dell'età adolescenziale; dall'altro, dall'alto grado di esposizione a nuove forme di dipendenza della popolazione giovanile. Per questa giovanissima coorte spesso internet - e, per associazione, tutti gli strumenti che permettono l'accesso al mondo virtuale, in primis lo smartphone - non è un semplice strumento comunicativo, ma una vera e propria appendice del proprio corpo.

La rete ha definito nuove e più semplici modalità di approcciarsi alla vita quotidiana nelle sfere relazionali, famigliari e sociali. Ma accanto alle comodità innegabili si rende necessario sottolineare il lato oscuro della situazione. Si fa cenno solo brevemente alla sempre maggiore incapacità dei più giovani di affrontare conversazioni faccia a faccia, di stare da soli e praticare l'introspezione - attività invece fondamentale per prendere coscienza del proprio io (S. Turkle, 2016) - la prevalenza di forme di individualismo marcate e tendenze narcisistiche, per cui la propria vita deve essere messa in vetrina in maniera autocelebrativa, e la scarsità delle relazioni sociali, per di più mediate dalla rete e dai social media. Tutto questo sommato ai tratti di personalità tipici dell'adolescente, quali impulsività e ricerca di sensazioni, o la scarsa tolleranza della noia, situazioni a cui le nuove tecnologie comunicative sembrano l'antidoto perfetto: attraverso lo smartphone il giovane accelera la sua esperienza del reale ed è bombardato da stimoli molteplici, esaudendo la necessità di immediatezza dei giovani. Non solo, anche l'ambiente famigliare, oggi spesso carente dal punto di vista educativo e poco in grado di definire regole e valori stabili, accompagna l'adolescente a ricercare modelli di riferimento nella costruzione della propria identità in realtà virtuali accessibili sul web (E. Caretti e D. La Barbera, 2015).

\section{Le new addiction: la dipendenza da tecnologie}

Nel panorama contemporaneo, i rapidissimi cambiamenti degli stili di vita, l'esiguità e la fluidità delle relazioni, la precarietà del mondo sociale e dei modelli culturali vigenti, hanno determinato nuove forme di disagio psicologico direttamente correlate ai nuovi strumenti di comunicazione. L'osservazione di tali fenomeni, ha permesso di introdurre un nuovo quadro di dipendenze patologiche comportamentali definite New Addiction, fra cui le dipendenze tecnologiche trovano posto.

Le New Addiction determinano una compromissione della vita dell'individuo nelle dimensioni sociale, relazionale, famigliare, economica e lavorativa. Si manifestano in modalità differenti in base allo specifico oggetto di dipendenza, pur condividendo caratteristiche psicopatologiche e sintomi comuni fra loro e con le dipendenze da sostanza - tolleranza, astinenza, investimento di tempo sempre maggiore nelle attività additive, interruzione delle normali attività a favore dei comportamenti di dipendenza, conflitti intra-psichici e con gli altri, ricaduta e craving (E. Caretti e D. La Barbera, 2015). Le New Addiction sono date dal sovrapporsi delle dimensioni individuale, sociale, culturale, relazionale e famigliare di un individuo: l'entità multifattoriale delle nuove dipendenze comportamentali rende quindi necessario approcciarsi ad esse in maniera multifocale e multidimensionale, in modo da indagare tutti i processi che le hanno originate e poter costruire un trattamento volto alla recovery (L. Pasqualotto et al., 2020). È su questa falsariga che nel 2001, OMS ha messo a punto un modello diagnostico incentrato sulla persona definito paradigma biopsicosociale (Classificazione Internazionale del Funzionamento, della Disabilità e della Salute, ICF), in grado di valutare non solo la dipendenza, ma l'intero funzionamento dell'individuo nelle molteplici dimensioni della sua vita. Il modello proposto da ICF è focalizzato sull'individuo, sulla sua percezione della malattia e della cornice che ne ha determinato l'insorgenza, sul suo contesto famigliare, i suoi vissuti e sfera emotiva. In tal senso, il trattamento non prevede il semplice contrasto della dipendenza, ma la risoluzione di tutte quelle situazioni, eventi, e condizioni che hanno determinato l'insorgere della malattia. Solo affrontando il problema alle sue radici e nelle sue molteplici direzioni, infatti, sarà possibile fronteggiare con successo le nuove dipendenze (L. Pasqualotto et al., 2020).

Fondamentale è la definizione di dipendenza patologica, cioè comprendere la sottile linea di confine fra comportamenti che necessitano una terapia e comportamenti che, tutto sommato, consentono di proseguire funzionalmente nella propria vita quotidiana. Il passaggio da normale a patologico scaturirebbe nel momento in cui il soggetto, pur di seguire l'oggetto di dipendenza - nel nostro caso specifico, un mezzo di comunicazione tecnologico subisce un appiattimento tale da privare la realtà del suo reale valore e una compromissione grave del funzionamento in aree importanti della sua vita. È necessario quindi individuare quei fattori di rischio e di protezione che possono determinare la condizione di dipendenza patologicamente detta o meno. Se da un lato è vero che mancanza di sostegno da parte dei caregivers o una relazione conflittuale con essi, presenza di tratti di personalità quali impulsività e ricerca della sensazione, condizioni di svantaggio sociale, economico e culturale, possono spingere il soggetto verso una condizione di dipendenza patologica; dall'altro è importante osservare come i fattori di protezione aumentino la resistenza e la resilienza, concorrano al mantenimento dello stato di benessere, e siano in grado di ridurre, compensare e neutralizzare i fattori di rischio precedentemente illustrati (V. Lingiardi e F. Gazzillo, 2014). Sono esempi di fattori di protezione positivi la presenza di figure di riferimento e famigliari positive, con stile educativo supportivo bilanciato fra sostegno e autorevolezza, un gruppo di pari popolato da figure non trasgressive, e la presenza di una buona rete sociale di supporto, che fornisce una vera e propria rete di sicurezza personale contro lo sviluppo della dipendenza patologica.

Le dipendenze da tecnologie sono definibili disturbi tecnomediati che vedono un utilizzo eccessivo e compulsivo delle tec- 
nologie della comunicazione, con gravi compromissioni sul funzionamento di importanti dimensioni della vita quotidiana, sociale, relazionale, famigliare, lavorativa e accademica, economica (E. Caretti e D. La Barbera, 2015). I casi più gravi vedono modificazioni disfunzionali dello stile di vita e comportamenti di assoluto isolamento nel proprio rifugio virtuale, utilizzato per neutralizzare le ingestibili emozioni negative. La dipendenza tecnologica crea un rapporto preferenziale del soggetto con lo strumento tecnologico e un graduale spostamento verso il mondo virtuale, che sostituisce per importanza quello reale.

Rispetto alla dipendenza da tecnologie, il soggetto patologicamente dipendente vive il bisogno irrefrenabile di utilizzare le tecnologie comunicative, immergendosi nel mondo virtuale a tal punto da evitare quello reale. Durante le sessioni, sempre più frequenti e lunghe, perde il senso del tempo e dello spazio, e si trasferisce mentalmente nella dimensione virtuale, da cui è sempre più difficile uscire. Può inoltre giustificare il proprio comportamento adducendo a motivi di studio o lavoro che giustifichino l'uso intensivo dello strumento tecnologico, quando in realtà vive una totale mancanza di controllo sul proprio comportamento (G. Lavenia, 2018).

Dall'uso additivo, le interferenze con il funzionamento del soggetto riguarderanno soprattutto le seguenti dimensioni:

- Relazionale e famigliare: l'aumento progressivo del tempo impiegato nelle sessioni online diminuisce quello dedicato alle persone significative e alla famiglia. L'immersione totale nel virtuale spinge il soggetto a prediligere le interazioni online a scapito di quelle reali, alimentando l'isolamento sociale.

- Lavorativa e scolastica: il gran tempo non necessario dedicato al mondo virtuale danneggia il soggetto in ambito lavorativo e accademico, che non solo dispone di meno tempo per completare i propri compiti, ma sarà anche danneggiato nella memoria e nell'apprendimento, a causa della deprivazione di sonno che l'uso eccessivo delle tecnologie induce.

- Salute: danneggiamento a livello físico del soggetto, con problemi di vista, disturbi del sonno, irregolarità dei pasti, mal di schiena e mal di collo, mal di testa.

- Economico-finanziario: lo scadere della performance lavorativa ha conseguenze negative sulle entrate economiche dei soggetti. Non solo, le dipendenze tecnologiche quali trading patologico online o gioco d'azzardo patologico online impattano direttamente sul portafoglio della persona, spinta dall'impulso irrefrenabile di scommettere e giocare in attesa della grande vittoria.

Nella seguente analisi si farà riferimento specifico alla dipendenza da social network o Social Networking Sites (SNS) Addiction. La dipendenza da social network è una sottocategoria dello spettro della IAD - Internet Addiction Disorder, cioè un comportamento di utilizzo problematico della rete internet, che fa spendere parecchio tempo, con sessioni sempre più lunghe e frequenti, è causa di stress, e produce una compromissione e una menomazione forte e disfunzionale nelle principali aree esistenziali - personale, relazionale, scolastica, familiare e affettiva (M. Shaw e D. Black, 2008).

Similmente, la SNS Addiction è uno stato psicologico disadattativo di dipendenza nell'uso di un social network, che si manifesta attraverso un pattern ossessivo di controllo e uso della piattaforma, tale da alterare il normale funzionamento, e produrre una serie di sintomi comportamentali tipici delle dipendenze -salienza, tolleranza, conflitto, recidiva e astinenza. Alla pari della dipendenza da internet, la sintomatologia comprende alterazioni dell'umore, cognitive e delle reazioni fisiche ed emotive; problemi interpersonali e psicologici; aumento dello stress, dell'ansia e depressione (H. Hou et al., 2019; I. Pantic, 2014). Nonostante sia un terreno di ricerca relativamente nuovo, e ad oggi ancora non indagato in tutte le sue dimensioni, è stato possibile identificare la relazione positiva fra dipendenza da social e danneggiamento della salute mentale, della performance accademica e lavorativa, della vita sociale e del benessere individuale (O. Calancie et al., 2017).

Essendo i social network piattaforme utilizzate da un numero elevatissimo di persone - Hootsuite ha calcolato che, a inizio 2021, gli utenti dei social media sarebbero stati 4.2 miliardi in tutto il mondo (Hootsuite, 2021) - si rende fondamentale mettere in luce la differenza fra coloro che soffrono di dipendenza e i soggetti che semplicemente fanno un uso massiccio dei social: i primi, infatti, svilupperebbero un utilizzo incontrollabile e compulsivo delle piattaforme, che porta a conseguenze indesiderabili e dannose per la salute; sono individui coinvolti in maniera esagerata nello spazio virtuale, e che vivono un'urgenza incontrollabile di accedere e utilizzare gli spazi sociali virtuali.

I fattori determinanti nelle dipendenze da social media sarebbero tre:

- Il confronto sociale è un elemento che media la relazione fra utilizzo dei social e benessere individuale. La teoria del confronto sociale (L. Festinger, 1984) è uno dei meccanismi chiave per spiegare le conseguenze emotive e psicologiche dell'abuso delle piattaforme sociali. È definibile come la tendenza a utilizzare gli altri come fonti di informazione, per poter determinare, sulla base del paragone, il proprio livello di abilità o in che modo dovrebbero comportarsi, pensare e sentirsi rispetto alla maggioranza. Sulla base delle informazioni raccolte, obiettivo del confronto è consentire al soggetto di muoversi tranquillamente nel mondo sociale. Il paragone, inoltre, è più impattante se l'altro è simile a noi, e tendenzialmente avviene dal basso verso l'alto, ossia con la percezione dell'altro come migliore. Questo spiega perché sui social - più che nei media tradizionali - il confronto sociale è una tendenza così forte: la maggioranza degli utenti sono persone normali a cui naturalmente tendiamo a paragonarci, ma che ci bombardano di informazioni strumentalmente manipolate per fornire la migliore immagine possibile di sé. Il confronto diventa così un fattore di vulnerabilità, e può determinare una riduzione dell'autostima, percezione di inadeguatezza, e maggiori livelli di depressione e solitudine (P. Verduyn et al., 2020).

L'autostima è l'elemento che media la correlazione positiva fra abuso degli spazi sociali e disturbi mentali. Il costante confronto con ciò che gli altri utenti condividono e con i loro successi, la distorta percezione fisica, emotiva e sociale propria e degli altri, i sentimenti di gelosia e i comportamenti individualistici e narcisistici, sono fattori che possono impattare sul livello di autostima individuale. I soggetti, infatti, spesso utilizzano le piattaforme sociali per mettersi a confronto con le levigate e glorificate immagini di sé prodotte da altri utenti, in un confronto sociale dal basso verso l'alto che produce un abbassamento dell'autostima, dietro la convinzione che le vite degli altri utenti siano più felici e di maggior successo rispetto alla propria. La dipendenza da social abbassa i livelli di autostima individuale, e bassi livelli di autostima sono correlati a cattiva salute mentale, in particolare a disturbi quali depressione, dipendenze e disordini alimentari.

Infine, il bisogno di gratificazione psicologica: giorno per giorno il soggetto ha necessità di soddisfare una serie di bisogni psicologici fondamentali - competenza, autonomia e relazione. La dipendenza da social network si realizza nel momento in cui l'utente si convince che tali bisogni sono realizzabili solo nello spazio virtuale: giovani con bassi livelli di soddisfazione dei bisogni potranno trovare più semplice entrare in contatto con l'altro e sviluppare competenze sociali nell'ambiente virtuale, percepito come rifugio dove saranno in grado di compensare $i$ propri deficit di soddisfazione nella vita reale (Hou H., et al., 2019; Pantic I., 2014).

Molteplici ricerche negli anni hanno messo in evidenza il lega- 
me fra l'abuso di SNS con disturbi d'ansia, dell'umore e disturbi di personalità. La relazione negativa fra uso delle moderne tecnologie comunicative e salute psicologica ha portato alla definizione degli iDisorders (L.D. Rosen et al., 2013), declinati nelle seguenti dimensioni.

Per quanto riguarda i disturbi dell'umore, esistono oggi diversi studi a sostegno della relazione fra depressione ed eccessivo texting, visione di video clip, video gaming, chatting, ed e-mailing. Questa particolare forma di depressione sembrerebbe essere trasmissibile attraverso un contagio emotivo e sociale che passa per i social network. Sulle piattaforme sociali online gli utenti sono costantemente connessi e virtualmente vicini l'uno all'altro, con la costante possibilità di trasmettersi la percezione di isolamento sociale. La condivisione di contenuti depressi, contagerebbe gli altri utenti alla depressione e veicolerebbe i loro contatti successivi - quali chat con partner e amici, segnate da termini più tristi, lentezza nella risposta - in una forma di depressione di seconda mano. L'analisi delle condotte dei soggetti legate a un uso eccessivo dei social network hanno permesso a Rosen e colleghi di determinare anche i sintomi clinici che predirebbero l'emergere di sei disturbi di personalità:

- Disturbo paranoide di personalità: i soggetti che abusano dei social hanno punteggi maggiori di ideazione paranoica rispetto al gruppo di controllo. Lo stesso è stato rilevato nei soggetti che fanno uso eccessivo dei messaggi di testo e nei soggetti con Internet Addiction, i quali sembrano soffrire di percezioni anormali della realtà.

- Disturbo schizoide di personalità, caratterizzato da introversione patologica e ritiro sociale, disinteresse o incapacità a intrecciare relazioni interpersonali. L'abuso dei social network costituirebbe un sostituto alle relazioni e interazioni umane.

- Disturbo antisociale di personalità, tradizionalmente associato al consumo eccessivo di contenuti televisivi e pornografici e abuso di videogaming violento, che accentuerebbero i tratti di aggressività e violenza del soggetto. La correlazione con l'utilizzo dei social si ha nelle condotte di esibizionismo e senso di diritto, emerse nell'atto di vendicarsi rispetto a commenti negativi, controllo degli aggiornamenti altrui per verificare se si stia parlando del soggetto, e richiesta di maggior supporto sociale rispetto a quello dato agli altri.

- Disturbo istrionico di personalità, i cui segni si ritrovano in relazione al fenomeno del sexting, cioè l'invio di messaggi di testo con contenuto sessuale, e alla condivisione sui social di stati e immagini sessualmente esplicite al fine di manifestare ricerca di attenzione e rassicurazione, e necessità di esprimere la propria sessualità.

- Disturbo di personalità narcisista: le ricerche hanno mostrato come i giovani appartenenti all'ultima generazione mostrino punteggi molto più alti nel Narcissism Personality Inventory rispetto alle coorti precedenti. Si potrebbe parlare di una vera e propria epidemia narcisistica, sorta in corrispondenza con la massiccia diffusione dei social network: il legame fra i due fenomeni è evidente andando ad analizzare i contenuti proposti sulle piattaforme sociali, dove prevalgono forme di espressione di autopromozione e l'utilizzo maggiore dei pronomi io e me. Uno degli emblemi dell'atteggiamento autoreferenziale e autoconfermativo dei giovani utenti è il selfie: scattarsi un selfie e pubblicarlo, infatti, rinforzerebbe l'egocentrismo e il desiderio di stare al centro dell'attenzione. I social network sembrano inoltre il terreno ideale ad alimentare le tendenze egocentriche dei più giovani e incoraggiare i comportamenti narcisistici, poiché consentono un livello di controllo massimo del proprio ambiente sociale virtuale e la manipolazione della propria immagine, biografia, status e lista di amici.

- Disturbo ossessivo compulsivo di personalità: la travolgente quantità di informazioni e comunicazioni messe a disposizione dalle nuove tecnologie influisce negativamente sul benessere dei soggetti affetti da questo disturbo, che cercano di inibire l'ansia in altre forme di compulsione quali lo shopping online compulsivo, o l'uso compulsivo di internet, del telefono cellulare e dei social network (L.D. Rosen et al., 2013).

Facebook è il social network sottoposto a maggiore e più attenta analisi per quanto riguarda i problemi di ansia fra i giovani utenti. Molto spesso i giovani soffrono di disturbi d'ansia caratterizzati da scarsa autostima, incapacità di problem-solving e desiderio di essere accettati, tutti elementi che li predispongono ad alti livelli di stress nell'uso delle piattaforme social.

La prima fonte di ansia sociale sembrerebbero essere le funzioni like e commento ormai presenti in tutti i social. L'approvazione di un contenuto pubblicato sul web è descritta come una sensazione molto piacevole da parte dell'utente, ma, allo stesso tempo, le emozioni positive scaturite dal consenso altrui sono spesso percepite come false ed effimere, prive di significato profondo. Al contrario, ricevere un feedback negativo o, ancora peggio, non ricevere alcun feedback, determina emozioni particolarmente negative. Alla base di questi stati emotivi c'è la tendenza al confronto con i propri pari, che crea forti sentimenti di ansia e spinge a un comportamento compulsivo di costante controllo delle amicizie e delle attività altrui. I social permettono di realizzare forme di confronto sociale senza precedenti, e maggiore è l'importanza data all'online social comparison, maggiore è l'impatto sul benessere degli utenti (O. Calancie et al., 2017).

La situazione è particolarmente grave per i giovani che già soffrono di disturbo di ansia sociale, e che spesso si rivolgono ai social per compensare la mancanza di relazioni faccia a faccia che garantirebbero loro supporto emotivo. Purtroppo, però, le piattaforme sociali virtuali hanno una capacità molto limitata di offrire il supporto sociale necessario, e, anzi, espongono gli adolescenti già fragili a un numero maggiore di stressor. Questo vale sia per giovani con bassa autostima e immagine di sé negativa - che cercano negli spazi virtuali online un terreno di approvazione sociale - ma anche per adolescenti che presentano tratti esagerati di perfezionismo, i quali cercheranno di elaborare contenuti per ottenere un giudizio sociale molto positivo, in linea con i loro inarrivabili standard di perfezione. In caso di reazione negativa, però, saranno colpiti da un brutale senso di inadeguatezza, che alimenterà la distorta immagine negativa che hanno di sé stessi.

\section{Il fenomeno degli online death games: la Blue Whale Challenge}

Gli aspetti di abuso e dipendenza da social network, le forme di disturbi d'ansia, dell'umore e di personalità correlate, i disagi emotivo, psicologico e sociale che si vengono a determinare, apparirebbero fondamentali per comprendere il fenomeno degli online death games. Il rischio maggiore, e la tendenza diffusa nell'opinione pubblica, sembrerebbero l'appiattimento di questi tragici giochi mortali a spiegazioni semplicistiche e che non tengono conto di quelle che sono le radici del fenomeno.

Gli online death games sono una fenomenologia criminale compresa nella cosiddetta zona grigia del crimine digitale, comprensiva di tutte quelle attività al limite dell'illegalità, caratterizzate da importante dannosità e dolorosità per le vittime. Si tratta di sfide mortali che coinvolgono giovani e giovanissimi che, incapaci di affrontare il travagliato periodo di cambiamento che è l'adolescenza, vivono gli atti di autolesionismo come un modo per sentirsi vivi e fisicamente reali, facendo appello a una sofferenza fisica che è molto più semplice da affrontare rispetto a quella mentale e psicologica che accom- 
pagna il passaggio verso la vita adulta (G. Lavenia, 2018). I giochi della morte online sono forme di spettacolarizzazione della morte e macabri rituali che sempre più adolescenti scelgono di intraprendere e immortalare con i propri telefoni cellulari. La diffusione delle sfide spesso è alimentata dai giovani stessi, che tramite i dispositivi digitali si inoltrano i link di collegamento al gioco, allargando il numero di partecipanti e la complessità del fenomeno. Partecipare al gioco è vissuto come rito di iniziazione, permette di entrare nel gruppo, dimostrare forza e coraggio, fino a raggiungere una percezione di onnipotenza in un mondo reale che ha perso gli spazi di socialità e molti punti di riferimento.

Fra i giochi estremi diffusi in rete, quello che ha ottenuto maggiore eco mediatico è sicuramente la Blue Whale Challenge. Il forte impatto che questa sfida mortale ha suscitato nell'opinione pubblica ha stimolato la realizzazione di diversi studi in merito che, anche grazie alle rivelazioni fornite dal suo creatore, può essere preso come modello esemplificativo degli online death games e delle suggestioni in grado di produrre nei giovani partecipanti.

La Blue Whale Challenge ha origine nell'universo social russo VKontakte nel 2013, per poi diffondersi nelle principali piattaforme in tutto il mondo, attraverso Twitter, Facebook, Instagram e Reddit.

Il suo ideatore, Philipp Budeikin, giovane studente di psicologia russo, arrestato e processato con l'accusa di istigazione al suicidio nel 2017, ha affermato in un'intervista al Saint Petersburg News di averla creata con lo scopo di liberare il mondo da coloro che "creano solo danni a loro stessi e alla società" (P. Budeikin, 2018). "Ci sono le persone e ci sono gli scarti biologici", ha spiegato nella stessa intervista," ci sono persone che non hanno alcun valore all'interno della società. Li ho spinti al suicidio per purificare la nostra società. [...] Non sono pentito si ciò che ho fatto, anzi. Un giorno capirete tutti e mi ringrazierete" (P. Budeikin, 2018).

La Blue Whale Challenge è una sfida fisica e mentale, artico- lata in una serie di prove che prescrivono comportamenti autolesivi. Coinvolge un gruppo online, i cui protagonisti sono una vittima, una serie di spettatori passivi, e un curatore, detto anche master, che controlla il gioco e ha il compito di sottoporre le prove. Attraverso il social network Reddit è stato possibile recuperare la presunta lista delle cinquanta prove, da svolgersi in cinquanta giorni, che compongono la Blue Whale Challenge (Fig. 1). Le sfide, con prove di intensità e dolore crescente e una graduale escalation verso forme sempre più macabre di autolesionismo, devono essere fotografate o videoregistrate per dar prova della loro realizzazione, e svolte nella massima segretezza. Il gioco si conclude con il suicidio della vittima che, a questo punto, vivrebbe uno stato di alienazione, completo distacco dalla realtà e bassissima percezione del rischio e del pericolo (B. Khattar et al., 2018). Attraverso l'analisi delle prove è stato possibile individuare i possibili meccanismi psicobiologici sfruttati dai curatori per assicurarsi il controllo delle vittime. Il master è infatti in grado di incitare all'auto-lesione e all'auto-mutilazione, fino a spingere la vittima al suicidio: l'iter manipolatorio è esercitato sulla vittima fino alla prova finale, sfruttando tecniche basate sulla psicologia della paura ed elementi di induzione, abitudine e autolesionismo (A. Kumar et al., 2017).

In primo luogo, le istruzioni date dal master devono essere interattive e saper sfruttare il sistema cerebrale della ricompensa: in altre parole, occorre indurre il cervello a percepire ogni azione autolesiva come un atto di coraggio e forza, così da stimolare la motivazione necessaria per accettare la prova successiva. Per fare ciò, il curatore costruisce le prove in modo graduale e le alterna a momenti di adescamento mentale, in cui utilizzerà contenuti quali musica, film, e video che inibiscano la naturale paura umana del1'auto-mutilazione. La gradualità delle prove consentirà al master di accrescere nel tempo la gravità delle mutilazioni e ridurre le esitazioni del partecipante. Ogni fase completata con successo accre-

\author{
1. Carve with a razor "f57" on your hand, send a photo to the curator. \\ 2. Wake up at 4.20 a.m. and watch psychedelic and scary videos that curator sends you. \\ 3. Cut your arm with a razor along your veins, but not too deep, only 3 cuts, send a photo to \\ the curator. \\ 4. Draw a whale on a sheet of paper, send a photo to curator. \\ 5. If you are ready to "become a whale", carve "YES" on your leg. If not, cut yourself many \\ times (punish yourself). \\ 6. Task with a cipher. \\ 7. Carve " $f 40$ " on your hand, send a photo to curator. \\ 8. Type "\#i_am_whale" in your VKontakte status. \\ 9. You have to overcome your fear. \\ 10. Wake up at 4:20 a.m. and go to a roof (the higher the better) \\ 11. Carve a whale on your hand with a razor, send a photo to curator. \\ 12. Watch psychedelic and horror videos all day. \\ 13. Listen to music that "they" (curators) send you. \\ 14. Cut your lip. \\ 15. Poke your hand with a needle many times \\ 16. Do something painful to yourself, make yourself sick. \\ 17. Go to the highest roof you can find, stand on the edge for some time. \\ 18. Go to a bridge, stand on the edge. \\ 19. Climb up a crane or at least try to do it \\ 20. The curator checks if you are trustworthy. \\ 21. Have a talk "with a whale" (with another player like you or with a curator) in Skype. \\ 22. Go to a roof and sit on the edge with your legs dangling. \\ 23. Another task with a cipher. \\ 24. Secret task. \\ 25. Have a meeting with a "whale." \\ 26. The curator tells you the date of your death and you have to accept it. \\ 27. Wake up at 4:20 a.m. and go to rails (visit any railroad that you can find). \\ 28. Don't talk to anyone all day. \\ 29. Make a vow that "you're a whale." \\ 30-49. Every day you wake up at 4:20am, watch horror videos, listen to music that "they" \\ send you, make 1 cut on your body per day, talk "to a whale." \\ 50. Jump off a high building. Take your life.
}

Fig. 1. Blue Whale Game Rules.

Fonte originale: Reddit. 
scerà nel giovane sicurezza e consapevolezza di possedere delle qualità caratteriali, forza, coraggio e capacità uniche.

$\mathrm{Al}$ tempo stesso il curatore dovrà mostrarsi sensibile e vicino ai partecipanti, fornirgli amicizia e supporto, motivandoli a intraprendere sempre nuovi rischi. L'obiettivo è diventare la figura di riferimento del gruppo, colmando il vuoto lasciato dagli adulti nella vita reale. Su questo aspetto lo stesso Budeikin ha confessato di essere riuscito a dare alle sue vittime tale calore, comprensione e importanza da renderle felici di togliersi la vita. Il contatto con il master porta infatti a una riduzione delle preoccupazioni legate al gioco e prepara gli adolescenti ad accettare il dolore delle minori ferite fisiche come sacrificio per poter completare la prossima grande sfida finale (A. Kumar et al., 2017). Il livello di confidenza raggiunge un'intensità tale che i partecipanti non solo eseguono le sfide proposte, ma sono anche disposti a condividere col curatore dati sensibili, quali e-mail e numero di telefono, così da poter assicurare una relazione costante.

Fattore ancora più importante è la capacità di manipolare il meccanismo di sopravvivenza primario della paura. Per assicurarsi che il gioco sia portato a termine è necessario che sia costruito come un'avventura, sfruttando contemporaneamente la psicologia della paura, in modo da bypassare le reazioni fisiologiche alla paura dei partecipanti, e la caratteristica della sfida insita negli adolescenti, maggiormente inclini ad esporsi ai rischi. Applicando le strategie terapeutiche di sovra-esposizione a stimoli di minaccia è possibile ridurre l'ansia e la paura di affrontarla. Superare la paura è percepito a livello cerebrale come avventuroso e psicologicamente gratificante, e sfidare un timore con successo determina piacere e soddisfazione. L'autolesionismo è una pratica naturalmente evitata legata alla paura per la sopravvivenza dell'organismo: compito del master è trasferire la percezione istintiva negativa dell'auto-mutilazione a una vittoria sulla paura.

Presentare le prove come parte di un gioco può alterare i meccanismi sinaptici e neuronali, fino a indurre il soggetto a percepire come gratificante la realizzazione di prove di livello maggiore. Una volta entrati nel circuito dell'avventura, uscire dalla sfida diventa sempre più difficile: ciò è emerso anche nelle interviste dei giovani che si sono presentati come giocatori sopravvissuti, i quali hanno sottolineato quanto, una volta giunti agli ultimi step, fossero loro i primi a non volersi arrendere e arrivare fino in fondo.

In accordo con quanto detto finora, Kumar ha proposto la distinzione delle prove in tre categorie, che creano diversi tipi di suggestione volti alla progressiva desensibilizzazione del soggetto (Kumar A. et al., 2017):

- Le prime nove sfide sono costruite in modo da motivare $\mathrm{i}$ soggetti con la tecnica dell'induzione, e comprendono task minori e relativamente poco pericolose.

- Fino alla venticinquesima, le prove si intensificano gradualmente, con la finalità di costruire senso di abitudine e impavidità, accrescere l'interesse dei partecipanti e diminuire i loro conflitti interiori. È proprio a conclusione di questa fase che sembra che il curatore riveli alla vittima quando dovrà compiere l'azione suicidaria.

- Gli ultimi giorni sono dedicati alla preparazione alla sfida finale, a cui le vittime giungono avendo ormai appianato quasi completamente le proprie paure e i propri istinti di sopravvivenza. Queste ultime prove, in particolare, sono realizzate per alterare il ritmo sonno-veglia della vittima, rendendo il giovane docile e completamente sottomesso alle disposizioni del master. Budeikin ha affermato, per spiegare il suo sistema di manipolazione, che "a un certo punto, è necessario spingere l'adolescente a non dormire la notte. In questo modo la loro mente diventa più suscettibile all'influenza" (Budeikin P., 2018).

Il master esercita su tutti i partecipanti un modello manipolato- rio mentale di gruppo (Lavenia G., 2018), con cui condiziona la vittima a portare a termine le prove, e rende omertosi i coetanei, che si limiteranno a osservare il giocatore senza intervenire nonostante la pericolosità dei comportamenti a cui assistono.

Gli spettatori passivi eserciteranno nella sfida il ruolo di giudice delle prove e del valore stesso della vittima, e avranno il compito di diffondere attraverso le piattaforme social le prove stesse. La vittima che riuscirà a proseguire più a lungo nella sfida sarà ben giudicata, e la sua figura esaltata nel mondo virtuale: il confronto positivo coi coetanei determinerà la riduzione dei conflitti interni al soggetto e lo spingerà a incorrere in rischi sempre maggiori. Il master farà anche leva sulla dinamica gruppale per assicurarsi che il giocatore ubbidisca e non abbandoni il gioco: gli spettatori esercitano infatti una fortissima pressione sociale sulla vittima in termini di riconoscimento e identità, anche attraverso la semplice osservazione.

La Blue Whale Challenge sembra in grado di determinare meccanismi psicobiologici predatori e autolesivi, rispettivamente nel curatore e nelle vittime. Gli adolescenti sarebbero le vittime perfette per questo tipo di aggressione, per via della loro struttura neuronale, ancora in formazione, e le scarse esperienze di vita. Lo studio della vittimologia ha permesso di identificare le categorie di giovani maggiormente propensi ad essere adescati e ingaggiare la sfida: si fa riferimento a giovani con situazioni famigliari problematiche, assenza di supporto emotivo, ed esperienze negative alle spalle quali rifiuto, abuso, fallimento, sconfitta sociale e mancanza di relazioni intime e significative. Fattore di rischio rilevante è anche la presenza di sintomi depressivi, isolamento, e tratti del disturbo borderline di personalità, che possono spingere i soggetti a desiderare la morte e ingaggiare la sfida per porre fine alle sofferenze della propria vita (A. Kumar et al., 2017).

\section{Online grooming: i parallelismi con i modelli di adescamento settario}

Il modus operandi attivato dal master per adescare le giovani vittime è molto simile alle strategie di suggestione utilizzate per indurre tensioni e disordini sociali, credenze superstiziose e comportamenti additivi. In questo caso, il meccanismo di influenza sarà protratto a distanza, motivando una fascia di popolazione vulnerabile e sovrasensibile attraverso uno specifico programma di manipolazione psichica (A. Kumar et al., 2017).

Il grooming è definibile come la tecnica di manipolazione psicologica utilizzata dagli adulti per superare le resistenze dei giovani e ottenere la loro fiducia. Si tratta di una tecnica utilizzata frequentemente nello sfruttamento sessuale dei minori, sia nel contesto online che offline. Per via delle sue caratteristiche manipolatorie, è facilmente identificabile anche nell'ambito dell'adescamento pro-giochi della morte.

In linea generale, il grooming della dimensione online si compone di cinque passaggi consecutivi:

1. Il tutto ha inizio con una prima fase di contatto, realizzato attraverso chat, gruppi, giochi online o richieste di amicizia sui social. Da qui si sviluppano le prime conversazioni con la vittima.

2. L'interazione si fa sempre più frequente e profonda per entrare nella fase della fiducia: obiettivo dell'adulto è conquistare l'adolescente, mostrarsi vicino e comprensivo, condividere le sue passioni, e supportarlo nel risolvere i suoi drammi quotidiani.

3. Conquistata la fiducia della vittima, ha inizio la fase di raccolta di informazioni. Gradualmente e con pazienza, si cercherà di ottenere tutte le informazioni a cui non ha accesso dal profilo social, quali le sue abitudini e quelle della famiglia, chi sono i 
suoi genitori, quali strumenti di comunicazione utilizza e se sono controllati.

4. La relazione fra adulto e minore diventa esclusiva e impenetrabile dall'esterno. Nei giochi della morte, determina la segretezza del percorso della giovane vittima e richiede un sempre maggiore isolamento rispetto ai suoi contatti del mondo reale.

5. La fase supplementare è quella del ricatto: se il giovane volesse infatti ritirarsi dalla relazione $\mathrm{o}$ dal gioco, l'adescatore potrà minacciare sia il giovane sia la sua famiglia grazie alle informazioni acquisite.

Una lettura attenta delle tecniche di grooming per la creazione dei gruppi della morte da cui si sviluppano i principali death games, può suggerire una vicinanza preoccupante con quei meccanismi di manipolazione psicologica utilizzati tradizionalmente nel mondo settario. Si presenteranno qui di seguito le analogie fra le tecniche di reclutamento settarie e i meccanismi di adescamento della Blue Whale Challenge.

La prima fondamentale tappa di alterazione del pensiero, messa a punto dalla setta per attirare nuovi adepti, è il reclutamento, che prevede l'utilizzo contemporaneo di tecniche di avvicinamento e tecniche di vendita con l'obiettivo di affascinare la vittima (G. Palermo e V. Mastronardi, 2005). Nella Blue Whale Challenge il primo contatto fra master e vittima avviene attraverso i social network seguendo due direzioni: può essere l'organizzatore del gioco a individuare le potenziali vittime; o possono essere gli utenti a cercare l'attenzione del moderatore attraverso post contenenti specifici hashtag o parole chiave, immagini e video per attirare la sua attenzione. Una volta compiuto l'adescamento, le conversazioni fra le parti sono per lo più realizzate in chat privata - uno dei mezzi preferiti è l'app di messaggistica istantanea Whatsapp - e, per tanto, difficilmente rintracciabili. Tramite l'analisi dei profili social, le informazioni condivise dagli utenti stessi e le conversazioni avute con essi, il moderatore valuta le potenziali vittime al fine di individuarne le caratteristiche e vulnerabilità psicologiche. Tale meccanismo replica sostanzialmente l'uso che i reclutatori delle sette fanno dei questionari di test di personalità per l'individuazione dei potenziali adepti. Nell'ambiente settario, seguono le tappe di avviamento alla dottrina, con l'obiettivo di far sviluppare nelle nuove leve una diversa percezione di sé e della realtà, e di radicamento nel gruppo (G. Palermo e V. Mastronardi, 2005). Un procedimento simile si registra sulla rete: il master da inizio a quella progressiva serie di prove con cui stimola nel soggetto una nuova percezione di sé stesso più forte, coraggiosa e capace di azioni che non si riteneva in grado di affrontare. Sempre col benestare del curatore, inoltre, va a crearsi la dinamica gruppale fra pari che sarà utile a generare pressione sociale e influenza, fino a ingabbiare i giocatori nella sfida mortale.

La quarta fase consiste nell'allontanamento dalla società e nell'isolamento fisico e affettivo. Nell'online grooming l'isolamento fisico non può attuarsi chiudendo il soggetto all'interno di una comunità, ma riesce a prodursi grazie alla segretezza e alla riservatezza richiesta alle giovani vittime, che si trovano sempre più isolate rispetto alla famiglia e ai pari, fatta eccezione, ovviamente, per i membri del gruppo della morte. L'alterazione nella vita affettiva si sviluppa invece nell'esclusività della relazione con il curatore, che si fa insostituibile mezzo di sostegno e supporto dei giocatori, confidente e amico, sostituendosi alle altre figure importanti della vita dell'adolescente. Dall'isolamento, si passa all'indottrinamento, realizzato attraverso una serie di tecniche che rendono molto complesso uscire dalla comunità settaria. Lo stesso meccanismo di ingabbiamento lo abbiamo osservato nei paragrafi precedenti, evidenziando come, una volta entrati nel gioco, per i giovani sia molto complesso uscirne. Spinti in primo luogo da motivazioni interne, determinate dall'iter di manipolazione psicologica cui sono conti- nuamente sottoposti, ma anche da meccanismi di ricompensa - per cui ogni sfida superata è un successo e motivo di esaltazione da parte dei compagni - e di punizione/minaccia, con cui il master tiene sotto controllo i giocatori che, a un certo punto, potrebbero decidere di voler abbandonare il gioco.

$\mathrm{Si}$ instaura, a questo punto, l'ultima fase, di mantenimento e rafforzamento della dottrina. Anche qui avremo il combinato utilizzo di tecniche positive - quali aproblematicità e supporto psicologico costante - e negative, come il ricatto e la minaccia (G. Palermo e V. Mastronardi, 2005). Nel corso della death challenge questa fase di manipolazione si intreccia con la precedente, con l'aumento progressivo non solo della crudezza delle prove, ma anche della profondità del legame col curatore e il gruppo. Il rafforzamento della dottrina è funzionale al punto da portare il giocatore ad accettare la sfida finale con distacco e piena adesione alle disposizioni del curatore.

Un ulteriore elemento di somiglianza fra il mondo delle sette e quello della Blue Whale Challenge sono i caratteri psicologici delle vittime. Le caratteristiche identificate nell'adepto tipo riguardano la presenza di una condizione di disagio, quali lutti, difficoltà economiche, professionali o problemi di salute, e una serie di vulnerabilità psichiche, soprattutto disturbi psichiatrici, emozionali, bassa autostima, e scarsa capacità di comunicazione (G. Palermo e V. Mastronardi, 2005).

I caratteri di vulnerabilità dei giocatori sono sorprendentemente simili: i giovani più propensi ad entrare nella sfida presenterebbero una condizione di disagio famigliare ed esperienze negative passate, difficoltà relazionali, e fragilità mentali legate a depressione, isolamento e presenza di tratti tipici di alcuni disturbi psichiatrici e di personalità. Gli adolescenti che più facilmente cadranno nella trappola della sfida mortale sono caratterizzati da particolare fragilità, bassa autostima, insicurezza, isolamento ed emarginazione sociale. Provengono spesso da una situazione sociale e famigliare sfavorevole e sono privi di figure di riferimento e sostegno, ruolo che il master è lieto di occupare in cambio di devozione assoluta. Similmente, i soggetti predisposti all'adesione a un culto hanno fragilità emozionali e circa la propria autoefficacia personale, hanno subito un crollo dei valori e delle sicurezze famigliari, e cercano figure vicarie a quelle materne e paterne. Tratto comune sembra anche il desiderio di provare di possedere una forza e delle capacità che non si credeva di avere: da un lato abbiamo gli adolescenti, che superando con successo prove estreme possono sviluppare senso di invincibilità; dall'altro gli adepti, che cercano di compensare i vissuti di inferiorità con un complesso vicario di potenza e che, grazie all'indottrinamento, finiscono per sviluppare un senso di superiorità rispetto al resto della società grazie alla propria adesione al gruppo.

\section{Conclusioni}

Con la provocatoria prima legge della tecnologia, Melvin Kranzberg ha affermato che "La tecnologia di per sé non è né buona né cattiva. Non è nemmeno neutrale" (M. Kranzberg, 1986). A seguito di quanto presentato finora, sembra un'espressione decisamente forzata. D'altra parte, bisogna considerare la realtà dei fatti: le dipendenze tecnologiche, IAD e SNS Addiction incluse, sono disturbi ad oggi esclusi dal DMS-5; il fenomeno degli online death games risulta molto meno diffuso rispetto a quanto presentato dai mass media; la stessa Blue Whale Challenge è emersa per la prima volta grazie all'inchiesta della giornalista russa Galina Mursalieva, che solo in seguito si è scoperto aver la tendenza alla spettacolarizzazione dei suoi casi.

Alla luce di queste nuove evidenze, le parole di Kranzberg 
possono essere interpretate in maniera differente. Gli strumenti digitali, di per sé, non fanno né bene né male: tutto dipende da chi, come e per quali scopi vengono utilizzati. L'analisi dell'impatto delle nuove tecnologie comunicative sul benessere individuale dovrebbe quindi aprirsi alla spiegazione di quali processi i nuovi media siano in grado di attivare, e gli effetti negativi o positivi rispetto alla dimensione individuale e al contesto culturale e sociale in cui si sviluppano. Allo stesso tempo è innegabile che le nuove tecnologie, così intrecciate alla nostra vita quotidiana e a prescindere dall'uso che ne facciamo, abbiano un impatto sulle nostre menti. L'invito è quello di non accettare acriticamente le posizioni di chi vede solo il bianco o il nero di questi strumenti, ma accogliere le diverse possibilità e le opportunità, in futuro, di superare i limiti attuali.

Una sola condizione resta fondamentale: le tecnologie sono mezzi dotati di potere. Quando accettiamo la loro presenza nella nostra vita quotidiana, occorre sempre prestare attenzione a non consegnare completamente sé stessi alla loro rete, col rischio di esserne trascinati via. Meglio imparare ad utilizzare gli strumenti digitali piuttosto che lasciarsi usare da essi (S. Turkle, 2011; P.E. Ceruzzi, 2003).

\section{Bibliografia}

American Psychiatric Association (2014), DSM-5. Manuale diagnostico e statistico dei disturbi mentali, Quinta edizione, Traduzione italiana, Milano, Raffaello Cortina.

Belk, R. (2013), Extended self in a digital world, Journal of Consumer Research 40:477-500.

Blanco M., Trombetta M., (2019), Giovani internauti e viaggi senza ritorno: il fenomeno "Blue Whale Challenge". https://www. scienzeforensi.org/blog/index.php?id=gcgnx $70 \mathrm{y}$

Calancie O., et al. (2017), Exploring how social networking sites impact youth with anxiety: A qualitative study of Facebook stressors among adolescents with and anxiety disorder diagnosis Cyberpsychology: Journal of Psychosocial Research on Cyberspace 11:2.

Caretti E., La Barbera D. (2009), Le nuove dipendenze: diagnosi e clinica, Roma, Carocci editore.

Cavioni V., et al. (2012), Essere e apparire: le identità digitali costruite in rete, in O. Albanese, M.B. Ligorio, M.A. Zanetti (a cura di), Identità, apprendimento e comunità virtuali. Strumenti e attività on line. F. Angeli Editore.

Ceruzzi P.E. (2003), A history of modern computing, Cambridge, The MIT Press.

Hootsuite [Internet] (2021), Digital Trends 2021. https://www. hootsuite.com/pages/digital-trends-2021

Hou Y., et al. (2019), Social media addiction: its impact, mediation and intervention Cyberpsychology: Journal of Psychosocial Research and Cyberspace, 13:4.

Jewkes Y., Yar M. (2010), Handbook of Internet crime, Devon, Willan Publishing.

Kaplan A., Haenlein M. (2012), Social media: back to the roots and back to the future, Journal of Systems and Information Technology 14:101-4.

Khattar, B., et al. (2018), White or Blue, the Whale gets its Vengeance: A Social Media Analysis of the Blue Whale Challenge, https://arxiv.org/pdf/1801.05588.pdf

Kross, E., et al. (2021), Social Media and Well-Being: Pitfalls, Progress and Next Steps, Trends in Cognitive Sciences 25: 55-66.

Kumar A. et al. (2017), Psychobiological determinants of 'Blue
Whale Suicide Challenge' victimization: A proposition for the agency mediated mental health risk in new media age, PsyArXiv. doi:10.31234/osf.io/8xh92

Lavenia G. (2018), Le dipendenze tecnologiche. Valutazione, diagnosi e cura, Firenze, Giunti Psychometrics.

Lingiardi, V., Gazzillo, F. (2014), La personalità e I suoi disturbi. Valutazione clinica e diagnosi al servizio del trattamento, Milano, Raffaello Cortina Editore.

Palermo G., Mastronardi,V. (2005), Il profilo criminologico: dalla scena del crimine ai profili socio-psicologici, Milano, Giuffré Editore.

Pantic I. (2014), Online Social Networking and Mental Health, Cyberpsychology, Behaviour, and Social Networking 17: 652-7.

Pasqualotto L., Carozza P., Cibin M. (2020), ICF, salute mentale e dipendenze. Strumenti per la riabilitazione orientata alla recovery, Roma, Carocci Editore.

Przybylski A.K., Weinstein N. (2013), Can you connect with me now? How the presence of mobile communication technology influences face-to-face conversation quality, Journal of Social and Personal Relationships 30:237-46.

Rosen L.D., et al. (2013), Is Facebook creating “iDisorders"? The link between clinical symptoms of psychiatric disorders and technology use, attitudes and anxiety, Computers in Human Behavior 29:1243-54.

Rosen, L.D. (2011), Teaching the iGeneration, Educational Leadership, 68:10-5.

Shaw M., Black D. (2008), Internet addiction: definition assessment, epidemiology and clinical management, CNS drugs 22:353-65.

Tiggemann M., Anderberg I. (2020), Social media is not real: The effect of "Instagram vs reality" images on women's social comparison and body image, New media and society, 22: 2183-99.

Turkle S. (2011), Insieme ma soli. Perché ci aspettiamo sempre di più dalla tecnologia e sempre meno dagli altri, Torino, Einaudi Editore.

Turkle S. (2016) La conversazione necessaria. La forza del dialogo nell'era digitale, Torino, Einaudi Editore.

Verduyn P., et al. (2020), Social comparison on social networking sites, Current Opinion in Psychology 36:32-7.

\section{Sitografia}

Cyberlaws (2019), Dalla Blue Whale al Choking Game: le macabre "sfide della morte" online, https://www.cyberlaws.it/2019/bluewhale-choking-game/ (consultato Maggio 2021).

Il Post (2017), Cosa sappiamo del "Blue Whale", senza allarmismi inutili, https:/www.ilpost.it/2017/05/17/blue-whale-suicidi/ (consultato Maggio 2021).

Istituto per lo Studio delle Psicoterapie (2020), Blue Whale e Jonathan Galindo: quando dietro a un gioco si nasconde un disagio, https://www.istitutopsicoterapie.com/blue-whale-ejonathan-galindo-quando-dietro-a-un-gioco-si-nasconde-un-dis agio/ (consultato Maggio 2021).

La Repubblica (2021), Dalla Blackout Challenge all'Eye balling: le sfide della morte viaggiano sui social, https://www. repubblica.it/cronaca/2021/01/21/news/dalla_black_out_challan ge_all_eye_balling_le_sfide_della_morte_viaggiano_sui_social $-2 \overline{8} 36 \overline{6} 086 \overline{1} /$ (consultato Maggio 2021 ).

Saint Petersburg News (2016), La sincera confessione dell'amministratore del gruppo della morte (registrazione 
audio), https://saint-petersburg.ru/m/society/grachev/353694/ (consultati Maggio 2021).

The Times of India (2017), Meet the 22-year-old creator of the 'Blue Whale' death game, https://timesofindia.indiatimes.com/ india/meet-the-22-year-old-creator-of-the-blue-whale-deathgame/articleshow/59860662.cms (consultato Maggio 2021).

Correspondence: Monica Calderaro.

E-mail: calderaromonica6@gmail.com

Key words: Internet addiction; iGeneration; social media. Parole chiave: Internet addiction; iGeneration; social media. Palabras clave: Addición a internet; iGeneration; medios sociales.

Received for publication: 19 January 2021.

Accepted for publication: 22 February 2021.

This article is distributed under the terms of the Creative Commons Attribution Noncommercial License (by-nc 4.0) which permits any noncommercial use, distribution, and reproduction in any medium, provided the original author(s) and source are credited.

${ }^{\circ}$ Copyright: the Author(s), 2021

Licensee PAGEPress, Italy

Rivista di Psicopatologia Forense, Medicina Legale, Criminologia 2021; 26:613

doi:10.4081/psyco.2021.613 